\title{
Research on Non-Contact Weak Current Detection Technology
}

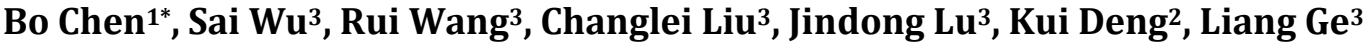 \\ ${ }^{1}$ College of Foreign Languages, Southwest Petroleum University, Chengdu, China \\ ${ }^{2}$ College of Electric and Information, Southwest Petroleum University, Chengdu, China \\ ${ }^{3}$ College of Mechanical and Electronic Engineering, Southwest Petroleum University, Chengdu, China \\ Email: *610500chenboswpu2019@163.com
}

How to cite this paper: Chen, B., Wu, S., Wang, R., Liu, C.L., Lu, J.D., Deng, K. and Ge, L. (2018) Research on Non-Contact Weak Current Detection Technology. Journal of Electromagnetic Analysis and Applications, 10, 67-75.

https://doi.org/10.4236/jemaa.2018.104005

Received: November 16, 2017

Accepted: April 27, 2018

Published: April 30, 2018

Copyright (C) 2018 by authors and Scientific Research Publishing Inc. This work is licensed under the Creative Commons Attribution International License (CC BY 4.0).

http://creativecommons.org/licenses/by/4.0/

\begin{abstract}
With the continuous development of industrial technology, the weak current plays an increasingly important role in all fields of life. In order to facilitate the user to carry, the study of contactless weak current measurement technology is also emerging. This article's design idea is based on two-dimensional reluctance sensor device built non-contact weak current detection system. The system uses the reluctance sensor HMC1002 to collect the current signal and the temperature sensor DS18B20 to compensate the temperature. The signals collected by the reluctance sensor and the temperature sensor are extremely weak. After being amplified by the amplifying circuit, the signal is conducive to subsequent detection and processing. Filter circuit can filter out interference signals to achieve the goal of improving accuracy. After the corresponding algorithm of the microcontroller will convert the signal voltage, easy to read and store, thus designing a non-contact current measurement capable of detecting weak currents and achieving higher accuracy.
\end{abstract}

\section{Keywords}

Weak Current, Reluctance Sensor, Non-Contact, Temperature Compensate

\section{Introduction}

In industrial production and scientific research, it is often necessary to measure the weak current flowing through a length of wire or conductor. Traditionally, the measured current is measured by flowing the current through a resistor of known resistance, measuring the voltage across the resistor, and then deriving the current from Ohm's law I = U/R. Due to the need to access the sampling resistor, not only the existence of the circuit under test to make the measurement 
results is difficult to accurately, and the test process is very inconvenient and difficult to automate.

In the study of a large number of references and journals, we found that the study of non-contact-type current testing techniques has been partially focused on the continuous improvement of the measuring circuit, and the other focus on new materials and new sensor technology. It's a different direction, but it's the same goal to increase the precision and reduce the cost of the device. So this subject uses a new type of reluctance sensor to measure the weak current, but the reluctance sensor is influenced greatly by the temperature, needing to introduce the temperature sensor, compensating the temperature influence through the software design, thus improving the survey accuracy.

Reluctance sensor using magneto resistivity, the current conductor and magneto resistive elements according to a certain combination of structures, when a current flows, the magnetic field generated by the current causes the magnetic resistance to change. This change can be detected by voltage signal, which can be detected in a certain current range [1]. Due to its small size, low power consumption, high sensitivity, make the magneto resistive sensor in the measurement and other technical aspects of more and more widely used. We want to design an instrument that can be very convenient to use the reluctance sensor to make contactless measurement of weak current. We want to contribute a new type of non-contact weak current measurement technology to completely isolate and insulate the measured circuit from the measuring circuit, and its sensitivity and precision are improved. Accurate measurement, good linearity, stable performance and good phase characteristics; since its load is basically tending to pure resistance, its output voltage phase is approximately the same as the measured phase. It also has the advantages of low cost, high cost performance, small volume, easy installation and debugging.

\section{Research Actuality}

All kinds of practical applications such as measurement and control of industrial production not only improve the accuracy requirement of weak current detection, but also put forward new requirements for detection methods. Therefore, the non-contact current detection system has been applied to a wider range of fields with its characteristics that are not affected by the measured circuit [2].

In recent years, the research of non-contact weak current measurement has been accelerated significantly and achieved remarkable results. The measurement accuracy is continuously improved and the performance is more stable. After looking up the data and reading the literature, it was found that the first use of inductance coupling between the circuits to realize the non-contact flow measurement, and now we use various magnetic detection sensors. At present, non-contact weak current measurement mainly adopts current transformer, coil, hall current sensor, optical current sensor, magneto resistance current sensor, etc. We know there are dozens of different working principle of current sensor, 
measurable range from $10^{-12}$ to $10^{-9} \mathrm{~A}$ [3], from dc to a few frequency of $\mathrm{MHZ}$, each sensor has the characteristics of their respective. In actual use, we can choose according to the need, and can even use two or more current sensors. Currently in non-contact weak current measurement research, on the one hand, adopt new technology and new materials, improve the sensitivity and accuracy of measurement, enlarge the application range, realize the miniaturization and integration, reduce weight and cost research high-performance sensors. On the other hand, it focuses on research and improvement of measuring circuit to improve detection accuracy [4]. We have found that the improved space in the measurement circuit is not large, while the use of high performance, low cost new sensors, and the use of a wide variety of sensor functions makes it easier to achieve increased sensitivity and precision of measurement and to reduce the cost of the actual industry demand.

\section{System Hardware Design}

The structure diagram of the whole system is shown in Figure 1.

Biaxial magnetic resistance sensor, working principle: under the influence of external magnetic field, the output voltage (OUT and OUT-) caused by the change of reluctance changes and that directly indicates the strength of the magnetic field. Owing to the device it does not need poly porcelain. It will not lead to hysteresis and repeatability. The chip has a built-in Honeywell patented current belt, which does not require an external coil. This type of sensor according to the four elements of a Wheatstone bridge configuration, which change the magnetic field into differential output voltage and can sense a magnetic field as low as $30 \mathrm{u}$ Gauss [5]. The sensor has the advantages of small size, low cost, high sensitivity, stabling reliability and so on. The specific internal and external structure of the HMC1002 is shown in Figure 2 and Figure 3.

The reluctance effect of reluctance sensor is the rule that the resistance value of $\mathrm{R}$ is changed along with the change of magnetic induction intensity $B$ when the conductive material placed in magnetic field is energized. The main principle is that the carrier of a conductive material is deflected by the Lorenz force in the applied magnetic field, resulting in a change in the resistance of the material. Its schematic diagram is shown in Figure 4. For the reluctance sensor, the external magnetic field is usually applied, which make the change of resistance value $(\Delta \mathrm{R} / \mathrm{R})$ and the resistance is in the Wheatstone bridge inside the reluctance sensor, thus causes the change of the voltage output of the bridge, that is, the output

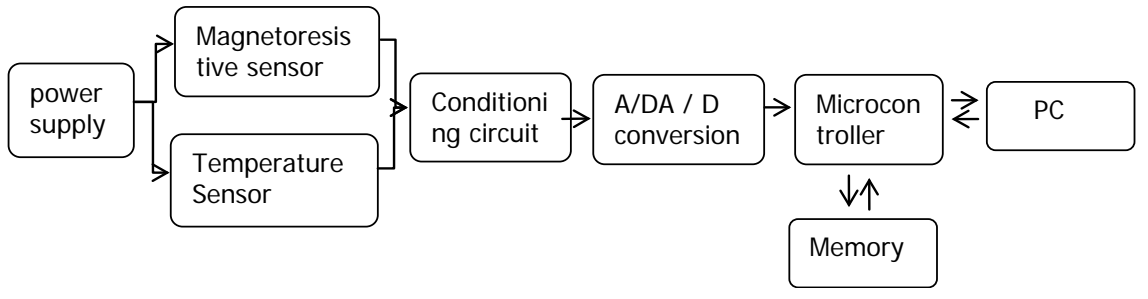

Figure 1. Overall block diagram of the system. 


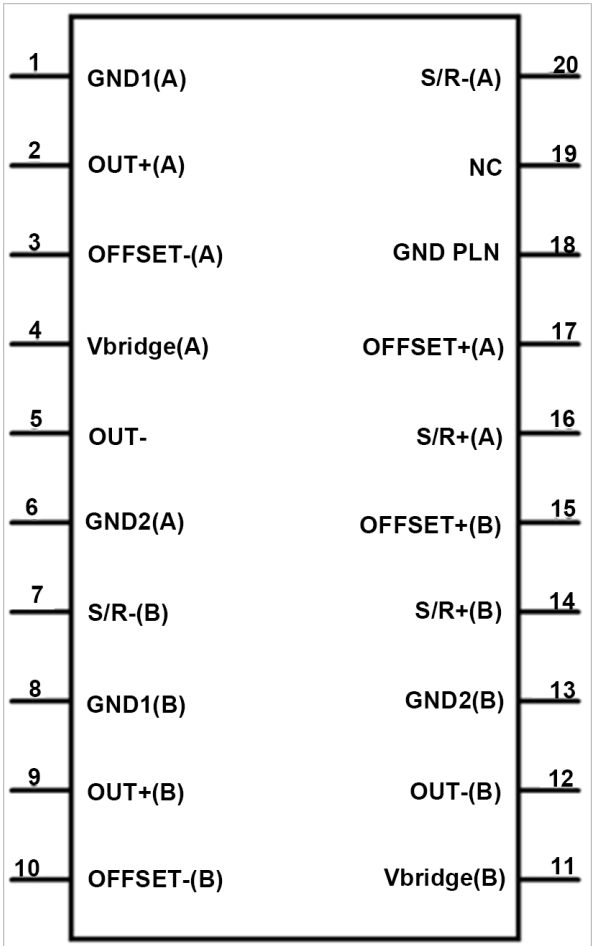

Figure 2. HMC1002 pin diagram.

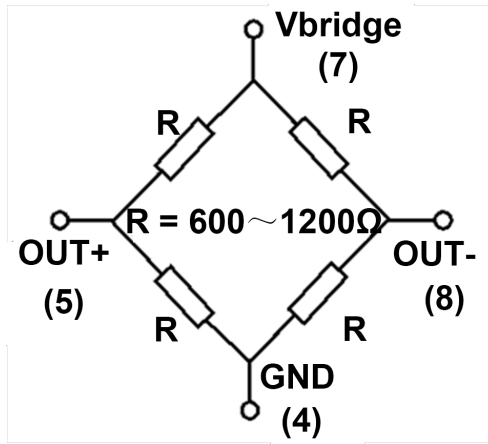

Figure 3. Internal circuit of HMC1002.
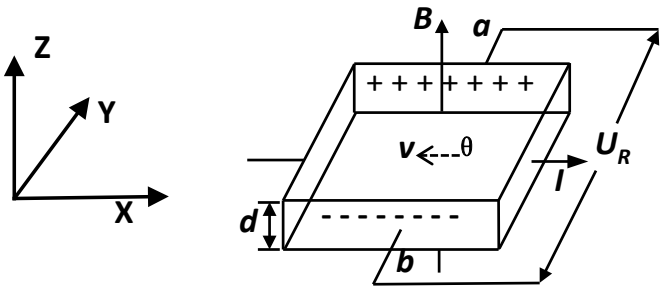

Figure 4. The principle of reluctance effect.

of the sensor is changed [6]. The resistance varies with the change of the external magnetic field, and causing the change of the output voltage is called the reluctance effect.

In magnetic field, the moving path of carriers in conductive materials will change due to the action of magnetic field. Generally, it will become longer. 
Increasing the electrical resistivity and increasing the resistance of conductive materials [7]. When the velocity of the two different carriers (electrons and holes) varies greatly, the resistivity of metal or semiconductor materials is mainly caused by the carrier with larger speed.

$$
\frac{\Delta \rho}{\rho_{0}}=0.273 v^{2} B^{2}=K v^{2} B^{2}
$$

Expression: $B$ is the magnetic induction intensity, $\rho$ is the resistivity of metal or semiconductor materials when the applied magnetic induction intensity is $B$, so $\rho_{0}$ is the resistivity of metal or semiconductor materials when the applied magnetic induction intensity is zero, $v$ is the velocity of the carrier of a conductive material in the magnetic field [8]. It can be seen from the first formula that when the magnetic induction intensity B is constant, the larger the carrier speed is, the more obvious the magnetoresistance effect is [9].

Digital temperature sensor, because this project adopts the method of temperature compensation to improve the measurement precision, which need to introduce the temperature acquisition module to collect the temperature [10] DS18B20 is a common digital temperature sensor, which has the characteristics of small volume, low hardware overhead, strong anti-interference ability and high precision. The temperature acquisition module is shown in Figure 5.

The intensity of the magnetic field produced by the weak current is small, so the voltage signals that output by the magnetoresistance sensor are also very weak [11]. It is necessary to add a signal amplification circuit to amplify the weak signal inconvenient detection and processing in the later period. AMP04 has the advantages of high precision, low power consumption, wide input voltage range. Excellent gain performance and so on, that can set the gain by an external resistance [12]. The gain range is 1 to 1000 meeting the actual requirements of this project, so AMP04 is used as amplifier to set up the amplifier circuit. The HMC1002 amplifying circuit is shown in Figure 6.

In the power circuit, connect A fuse of $13.2 \mathrm{~V} 0.5 \mathrm{~A}$ fuse to protect the circuit. When the current in the circuit is too large, the fuse can generate more heat, which can fuse the fuse quickly, thus protecting the whole circuit [13]. Setting the switch to control the circuit conveniently, the indicator light can determine whether the power circuit is connected. The power circuit is shown in Figure 7.

The communication circuit is mainly composed of the serial chip CH340 and the peripheral circuit, the $\mathrm{CH} 340$ is built with a separate transceiver buffer to support single, half-duplex, or full-duplex asynchronous serial communication. It provides the usual MODEM contact signal [14]. It is used to directly upgrade the common serial port equipment to the USB bus, and convert the output TTL level signal of the single chip computer into a USB interface to recognize the signal. The input and output of the chip is connected with the single-chip microcomputer, and the two-way communication between the computer and the single chip can be completed [15]. The communication circuit is shown in Figure 8. 


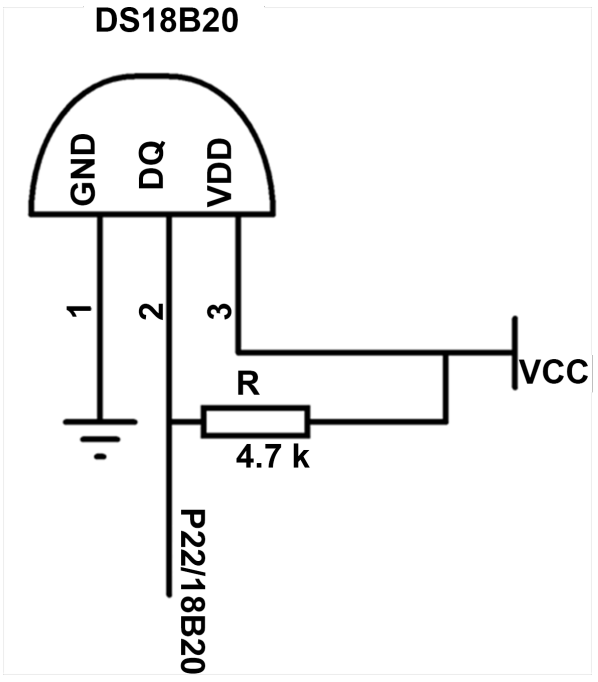

Figure 5. Temperature acquisition module.

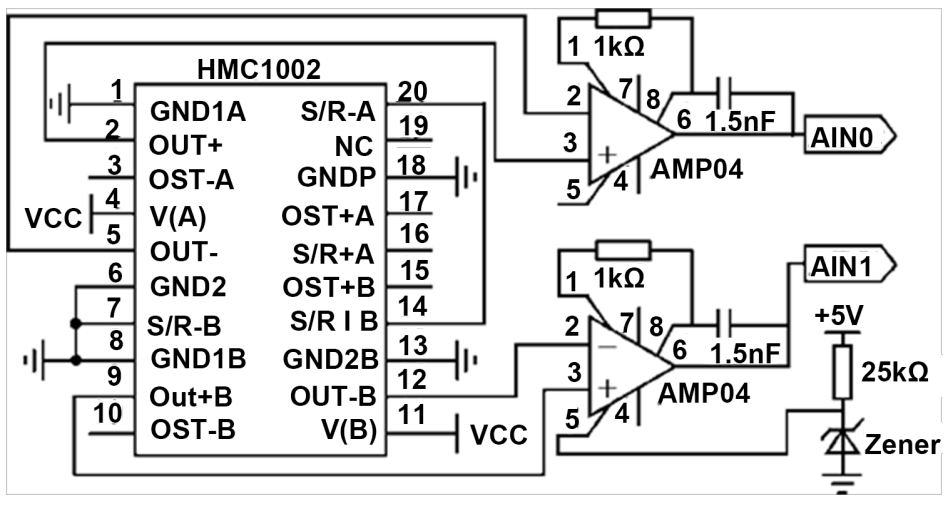

Figure 6. Signal amplification circuit.
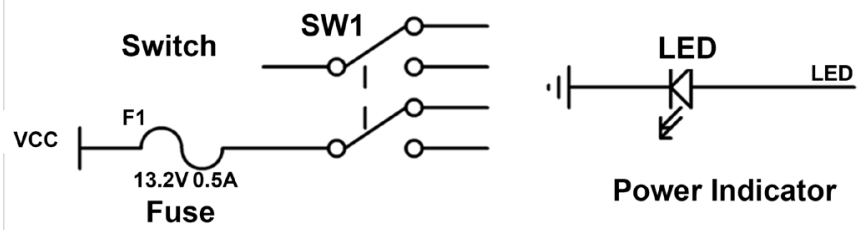

Figure 7. Power circuit.

\section{System Software Design}

On electric first, and then initialize the serial port, storage and so on to make it work at a predetermined work mode, and then begin to collect data cycle, the received temperature value and current value stored in the memory. The system software flow chart is shown in Figure 9 and Figure 10.

After setting up the test platform, connect the circuit. Debug and run the system's development board on the computer, measure experimental data. Then analyze the data, Calculation system error, and get the output characteristic curve of the system that can describe the performance of the system visually. The computer simulation test is shown in Figure 11. 


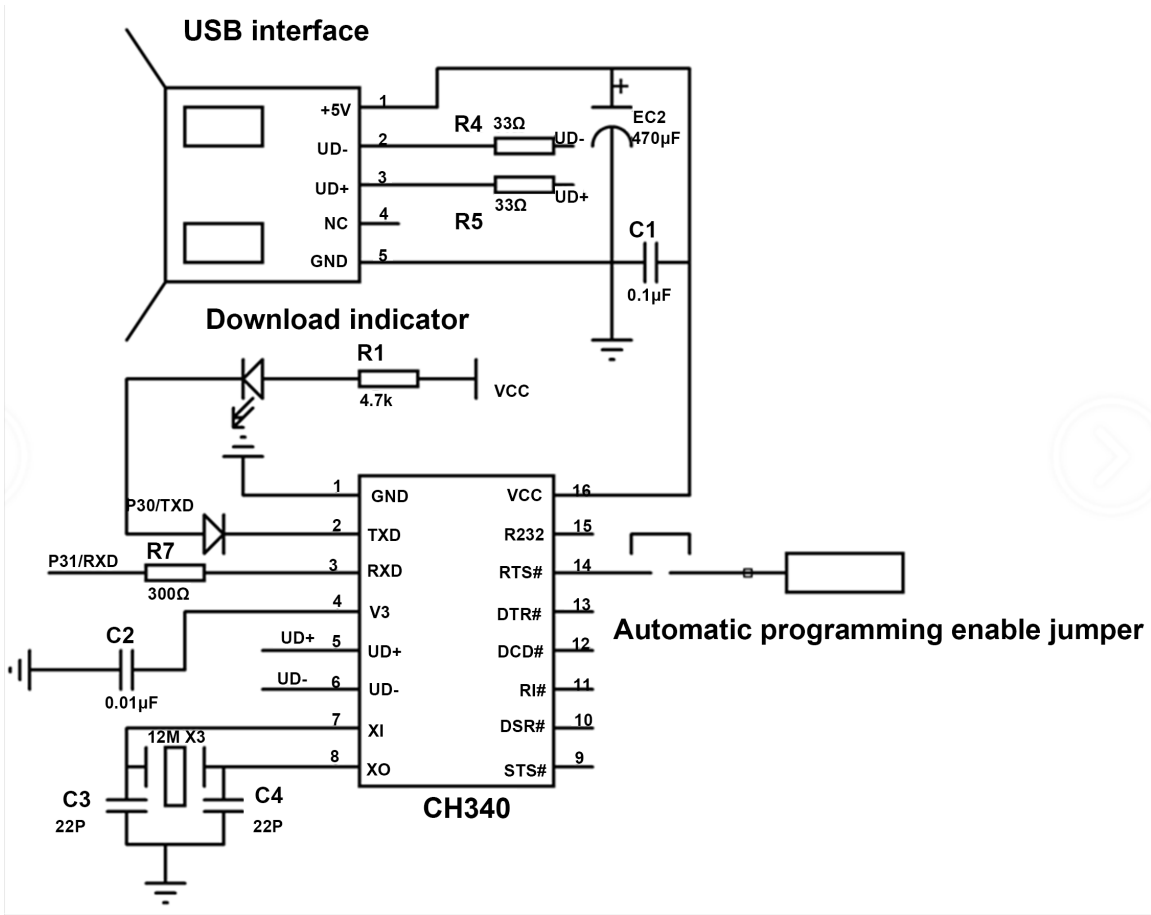

Figure 8. Communication circuit.

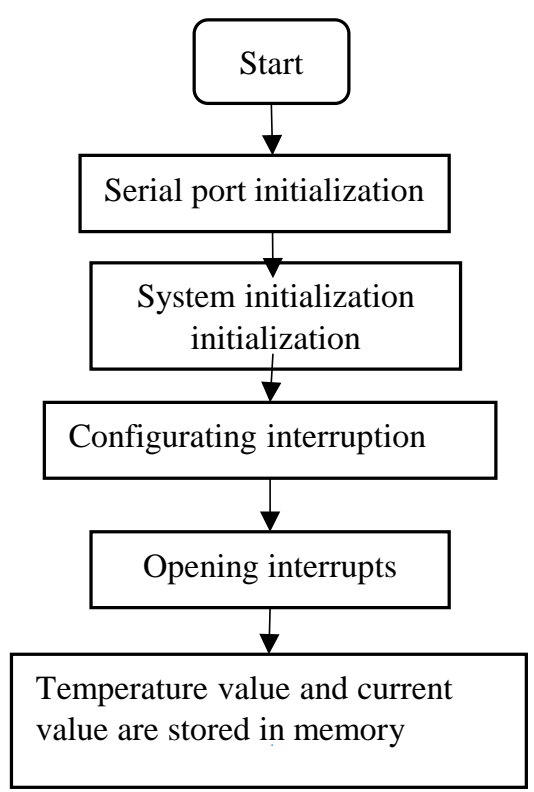

Figure 9. Main program flow chart.

\section{Conclusion}

This article in detail elaborates the non-contact two-dimensional magnetic resistance sensor weak electric current detection system research, and makes a practical measurement of the research. The system is analyzed objectively by combining theory and practice. According to the above experimental process and experimental time analysis, the whole system is feasible. The system uses 


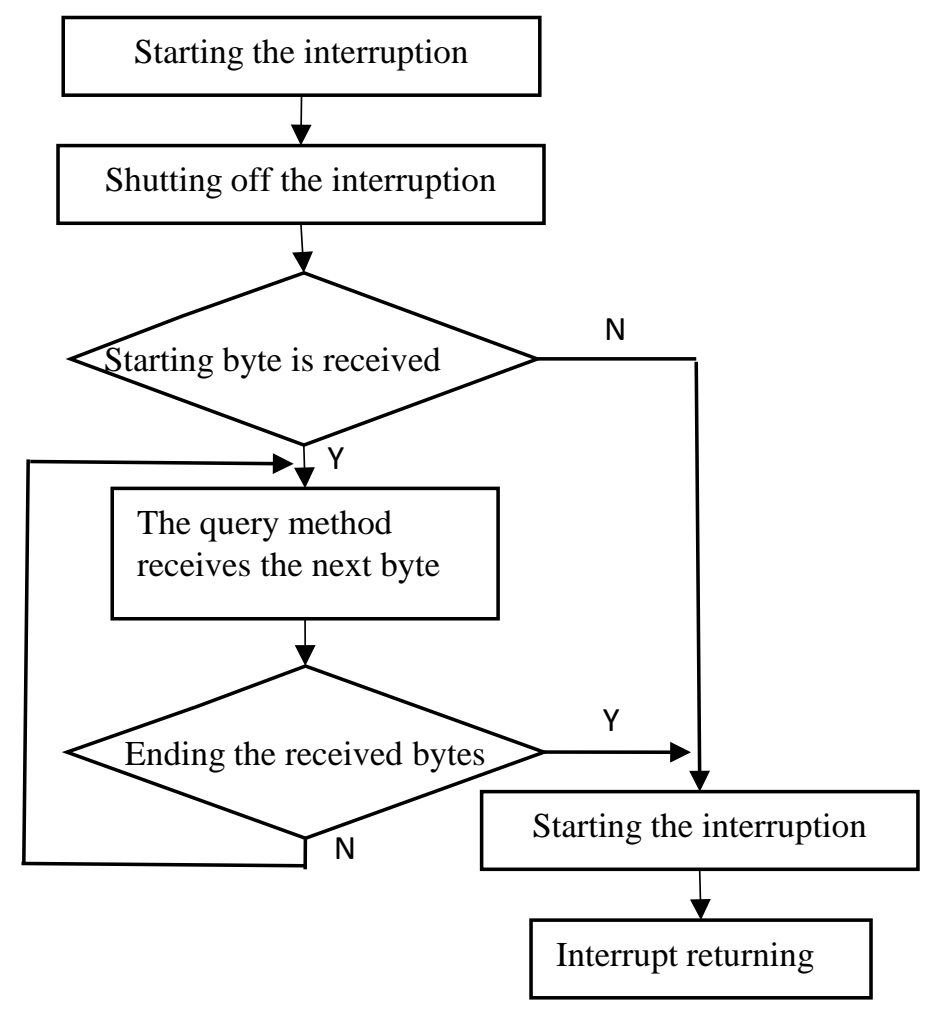

Figure 10. Sequence interrupt flow.

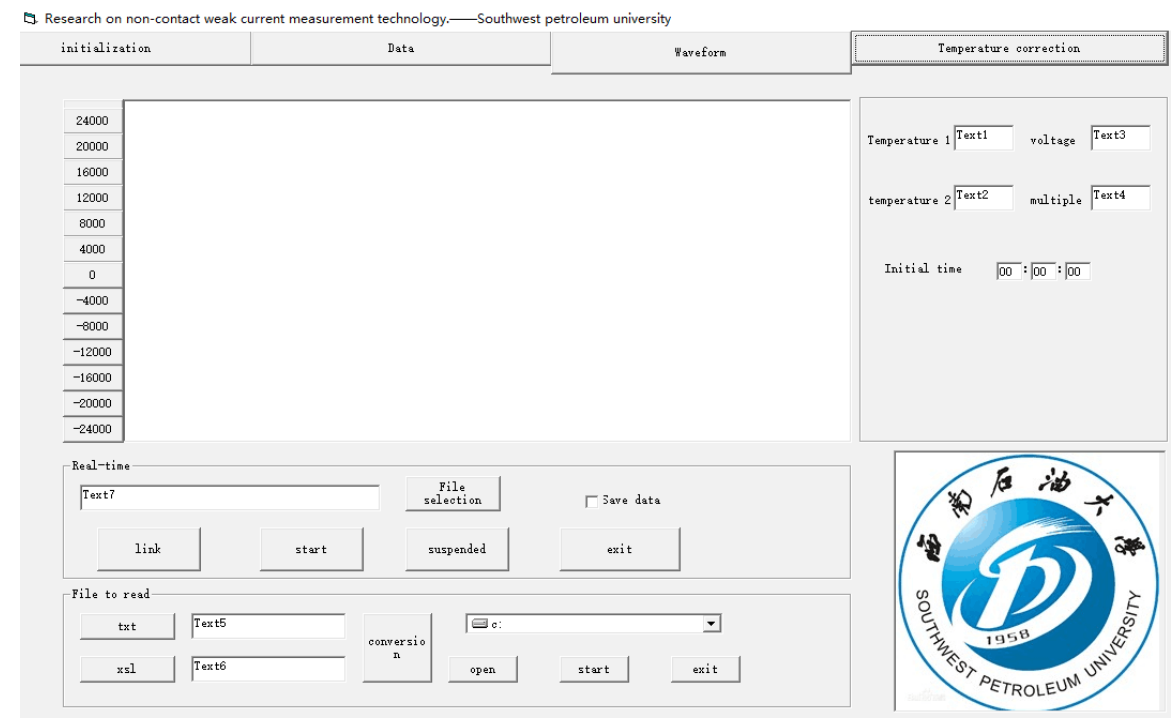

Figure 11. Computer simulation.

two-dimensional magnetoresistive sensor structure, HMC1002 itself magnetism gathering, so avoiding hysteresis and repetition of the phenomenon. The introduction of the temperature compensator eliminates the interference of experimental changes in ambient temperature to the experiment and greatly improves the experimental accuracy. Serial chip $\mathrm{CH} 340$ realizes two-way communication with computer and improves the system's fast processing performance. 


\section{Acknowledgements}

This work is Supported by the State administration of national security (No.sichuan-009-2013AQ, No.sichuang-0021-2014AQ,

No.sichuan-0011-2016AQ), the Sichuan educational committee (No.15ZB0060, 16ZB0082), the University student innovation industry project in China and Key project of College Students' open experiment in SWPU.

\section{References}

[1] Blazek, V. (2016) Ambient and Unobtrusive Cardiorespiratory Monitoring. 2016 ELEKTRO, Slovakia, 16-18 May 2016, 2. https://doi.org/10.1109/ELEKTRO.2016.7512022

[2] Texas Instruments (2001) TMS320F28x Optimizing C\&C++ Compiler User's Guide. Dallas.

[3] Texas Instruments (2003) TMS320F28x Analog to Digital Converter (ADC) Reference Guide. Dallas.

[4] Bratland, T. and Bilking, R. (2002) Selection and Application of Magnetic Sensors. Instruments and Sensors, 11, 18-21.

[5] Chase, C.R., Van Bibber, S. and Muniz, T.P. (2005) Development of a Noncontact Oil Spill Detection System. OCEANS, 2005. Proceedings of MTS/IEEE, Washington DC, 17-23 September 2005, 1352-1357.

[6] Cao, C.Z. and Wei, G.H. (2004) Optimization Design of Fuzzy Neural Network Controller in Direct Torque Control System. Proceedings of 2004 International Conference on Machine Learning and Cybernetics, Shanghai, 26-29 August 2004, 378-382.

[7] Yao, C.G., Wu, H., Mi, Y., Ma, Y., Shen, Y. and Wang, L. (2013) Finite Difference Time Domain Simulation of Lightning Transient Electromagnetic Fields on Transmission Lines. IEEE Transactions on Dielectrics and Electrical Insulation, 20, 1239-1246.

[8] Zhao, J., Gao, B., Woo, W.L., Qiu, F.S. and Tian, G.Y. (2017) Crack Evaluation Basedon Novel Circle-Ferrite Induction Thermography. IEEE Sensors Journal, 17, 5637-5645.

[9] Kiltz, S., Hildebrandt, M., Dittmann, J. and Vielhauer, C. (2012) Challenges in Contact-Less Latent Fingerprint Processing in Crime Scenes: Review of Sensors and Image Processing Investigations. 2012 Proceedings of the 20th European Signal Processing Conference (EUSIPCO), Bucharest, 27-31 August 2012, 1504-1508.

[10] Liu, P., Xiang, Huang, X. and Liu, F. (2006) From Simple to Smart: The Development of Sensor Network. Rare Metals and Engineering, 35, 617-620.

[11] Texas Instruments (2003) TMS320F2812 Digital Signal Processors. Dallas.

[12] Dumitru-Daniel, H.C. and Corodeanu, H.S. (2007) Magneto-Impedance Sensor for Biomedical Applications. Journal of Magnetism and Magnetic Materials, 1, 68-71.

[13] Li, L.J., Yuan, Y.S. and Feng, T.Z. (2006) Weak Signal Detection Based on Stochastic Resonance. Journal of Communications Technology and Electronics.

[14] Asai, T., Motoki,T, Gao, W., Ju, B.-F., et al. (2007) An AFM-Based Edge Profile Measuring Instrument of Diamond Cutting Tools. International Journal of Precision Engineering and Manufacturing.

[15] Sebald, G., Qiu, J. and Guyomar, D. (2005) Modelling the Lateral Resonance Mode of Piezoelectric Fibres with Metal Core. Journal of Physics D: Applied Physics, 38. https://doi.org/10.1088/0022-3727/38/19/023 